\title{
Rate of growth - a novel surrogate marker for high-risk cutaneous squamous cell carcinoma? A case report and review of the literature
}

Roberta Giuffrida ${ }^{1}$, Claudio Conforti², Karin Schmid ${ }^{3}$, Teresa Deinlein ${ }^{4}$, Iris Zalaudek ${ }^{2}$

${ }^{1}$ Department of Clinical and Experimental Medicine, Dermatology, University of Messina, Italy

2 Dermatology Clinic, Maggiore Hospital, University of Trieste, Trieste, Italy

${ }^{3}$ Institute of Physiology, Medical University of Graz, Austria

${ }^{4}$ Department of Dermatology and Venereology, Medical University of Graz, Austria

\section{Corresponding author:}

Dr. Roberta Giuffrida, MD

Department of Clinical and Experimental Medicine, Dermatology, University of Messina, Italy via Consolare Valeria ${ }^{\circ} 1,98125$ Messina, Italy

Telephone number: +390902212891

Email: roberta giuffrida@hotmail.it

Article type: Short Report

Short title: Rate of growth as marker for high-risk cSCC

Funding sources: none

Conflicts of interest: none declared

This article has been accepted for publication and undergone full peer review but has not been through the copyediting, typesetting, pagination and proofreading process which may lead to differences between this version and the Version of Record. Please cite this article as doi: $10.1111 /$ dth. 13156 


\section{Abstract}

Cutaneous squamous cell carcinoma ( $\mathrm{cSCC}$ ) is one of the most common non-melanoma skin cancer worldwilde, with a more invasive growth pattern and higher potential to metastatize than basal cell carcinoma. Although several risk factors have been linked to a high metastatic potential of cSCC, no widely accepted classification system for this common subtype of cancer exists. Herein we report an emblematic case of rapidly growing and metastatic cSCC and discuss the rate of growth of the tumour (ROG) as novel prognostic high risk surrogate marker.

Key words: non-melanoma-skin-cancer, squamous cell carcinoma, lymphoma-associated skin cancer 


\section{Introduction}

Cutaneous squamous cell carcinoma ( $\mathrm{CSCC}$ ) represents the second most common nonmelanoma skin cancer after basal cell carcinoma, with an increasing incidence worldwide. Surgical excision of the primary tumour is curative in the majority of cases, but about 4-5\% of patients will develop metastases. Metastatic cSCC is associated with severe morbidity and mortality. ${ }^{1}$ The 5 -year over all survival rate of metastatic cSCC is about $25 \%-35 \% .{ }^{2}$ Although several high risk factors have been linked to a high metastatic potential in cSCC, no generally accepted classification system is currently employed in this common subtype of cancer. ${ }^{3}$ It appears therefore crucial to identify specific tumour characteristics associated with an aggressive biology in order to develop better management and prevention strategies for this subgroup of patients.

\section{Case Report}

A 78-year-old Caucasian man was referred to our skin cancer unit because of a suspected diagnosis of cutaneous squamous cell carcinoma (cSCC) located on the right parietal-occipital scalp region (Fig. 1a). His medical history revealed several surgical interventions for actinic ratoses and early invasive cSCCs during the past 5 years. In addition, he suffered from a low-grade non-Hodgkin lymphoma not requiring treatment, hypertension, chronic renal failure, atrial fibrillation and implantation of permanent pacemaker. The patient was appointed for wide surgical excision 4 weeks later, but appeared just 8 weeks later. During this time, the tumour has increased significantly in size (Fig. 1b). Subsequent wide excision was performed and histopathological examination confirmed a moderately differentiated, 
ulcerated, infiltrative cSCC with neurotropism. The resection margins were tumour-free. A neo-adjuvant radiotherapy of the tumour bed and the draining lymph nodes was recommended, but he refused the treatment. Therefore close follow up visits were scheduled. Already at the first follow visit, just one month after the surgery of the primary tumour, a small, $6 \mathrm{~mm}$ in diameter measuring nodule was noted at the border of the scar (Fig. 1c). A recurrent cSCC was suspected and a wide surgical excision of the nodule was scheduled 4 weeks later. During this time, the tumour has significantly grown to a size of $2 \mathrm{~cm}$ (Fig. $1 \mathrm{~d}$ ). Subsequent wide excision with skin graft revealed a recurrent poorly differentiated cSCC with free margins. Only 2 weeks after the surgery of the recurrent cCSS, he developed a new, rapidly growing subcutaneous metastases at the right parietal-occipital region. The metastasis was surgically removed and the patient was lost for follow up. Six months later, he was referred because of a rapidly growing, ulcerated nodule localized on the right cervical region (Fig. 2a). Computer tomography examination revealed a mass of metastatic lymph nodes in the left cervical region. He was appointed for radiotherapy 10 days later. During these few days, the cervical metastasis has doubled in size and presented as painful, large and necrotic mass. (Fig. 2b). However, the patient once more did refuse radiotherapy and presented again two weeks later (Fig. 2c). Despite the increased severity of the disease, once again he denied any treatment and finally was lost to follow-up.

\section{Discussion}

cSCC represents the second most common non-melanoma skin cancer after basal cell carcinoma, with an increasing incidence worldwide. In particular, the term cSCC refers to a 
heterogeneous spectrum of malignant keratinocytic proliferations that differ with respect to their morphology, biology and attitude to metastasize. Surgical excision of the primary tumour is curative in the majority of cases, but about $4-5 \%$ of patients will develop metastases. Metastatic cSCC is associated with severe morbidity and mortality. ${ }^{1}$ The 5 -year over all survival rate of metastatic cSCC is about $25 \%-35 \% .^{2}$ Although several high risk factors have been linked to a high metastatic potential in CSCC, no generally accepted classification system is currently employed in this common subtype of cancer. ${ }^{3-4}$ It appears therefore crucial to identify specific tumour characteristics associated with an aggressive biology in order to develop better management and prevention strategies for this subgroup of patients. Recognised tumour-specific and patient-related high-risk features of cSCC, associated with an increased incidence of recurrence and nodal or distant metastasis include: (i) clinical diameter of $2+\mathrm{cm}$; (ii) growth in high-risk anatomic sites (peri-orifical sites, hands, feet); (iii) growth in embryonic fusion planes, in a chronic wound or burn scar, or on previously irradiated skin; (iv) local recurrence; (v) histopathologic thickness of 2+ mm; (vi) invasion beyond subcutaneous tissue; (vii) perineural invasion; (viii) poor histopathological differentiation, acantholytic or desmoplastic subtype, as well as (ix) host immunosuppression such as seen in organ transplant recipients or in patients with haematological co-morbidities. ${ }^{5}$ However, it is difficult to determine which risk factor has the greatest prognostic significance, because many of those can occur concurrently. As a consequence, there are no valuable prognostic models for high-risk cSCC.

Our case highlights a potential, not yet formally investigated, surrogate for high metastatic potential in cSCC, which is the rate of growth (ROG) (Fig. 3). Shortly, the ROG of a 3- 
dimensional tumour can be defined as the increase in tumour volume per unit of time. Notably, ROG is not an unknown tumour characteristic in dermato-oncology, as it is currently employed in classifying melanoma. ${ }^{6-7}$ The first recognizing ROG as important biological marker in melanoma was Clark et al. ${ }^{6}$ However, only since the landmark paper by Lipsker et al. ${ }^{8}$, in 2007, melanoma have been largely accepted to be subdivided into slow growing or fast growing subtypes with both types corresponding to specific epidemiological, morphological and metastatic characteristics. While studies mainly focused on the growth kinetics of melanoma growth and its implications for prognosis and management, there are currently very few studies assessing this aspect in the realm of $\operatorname{cSCC} .{ }^{9}$ Contrary to what we support with this report, Kricker et al. ${ }^{10}$ in their study about basal cell carcinoma and cSCC growth rates did not find an overall correlation between time and size of cSCC. However, two other reports described such positive association. ${ }^{11-12}$ Recently, Cañueto et al proposed a ROG of 4 $\mathrm{mm} /$ month, measured on H\&E-stained slides, as a reliable cutoff point for high risk cSCC, likely associated with poor outcome. ${ }^{9}$

Although the prognostic value of ROG in cSCC requires further research with a better assessment in larger prospective studies with adequate follow-up, our case highlights that cSCC, especially in the context of patients with haematological co-morbidities, should be equally managed as patients with fast growing, nodular melanoma, namely with prompt appointment for surgical excision without delay.

In conclusion, despite cSCC carries a low risk of metastases and death, it appears crucial to identify specific tumour and patients' characteristics associated with a more aggressive 
biology, such as the ROG of the tumour, in order to develop better management and prevention strategies. 


\section{References:}

1. Parikh SA, Patel VA, Ratner D. Advances in the management of cutaneous squamous cell carcinoma. F1000Prime Rep. 2014; 6:70. PubMed PMID: 25165569.

2. Kang SY, Toland AE. High risk cutaneous squamous cell carcinoma of the head and neck. World Journal of Otorhinolaryngology-Head and Neck Surgery, Volume 2, Issue 2, June 2016; 2:136-140.

3. Stratigos A, Garbe C, Lebbe C et al. European Dermatology Forum (EDF); European Association of Dermato-Oncology (EADO); European Organization for Research and Treatment of Cancer (EORTC). Diagnosis and treatment of invasive squamous cell carcinoma of the skin: European consensus-based interdisciplinary guideline. Eur J Cancer. 2015, Sep; 51:1989-2007. PubMed PMID: 26219687.

4. Conforti C, Giuffrida R, Pizzichetta MA, Di Meo N, Magaton-Rizzi G, Zalaudek I. Integrating the concept of field cancerization in the classification and risk assessment of cutaneous squamous cell carcinoma: proposal for a new classification and terminology of keratinocyte skin cancer. J Eur Acad Dermatol Venereol. 2019 Apr 13. doi: 10.1111/jdv.15624. [Epub ahead of print]. PubMed PMID: 30980765.

5. O'Bryan K, Sherman W, Niedt GW. et al. An evolving paradigm for the workup and management of high-risk cutaneous squamous cell carcinoma. J Am Acad Dermatol. 2013 Oct; 69:595-602. PubMed PMID: 23871719.

6. Clark WH Jr, From L, Bernardino EA, Mihm MC. The histogenesis and biologic behavior 
of primary human malignant melanomas of the skin. Cancer Res. 1969 Mar; 29:705-27. PubMed PMID: 5773814.

7. Liu W, Dowling JP, Murray WK et al. Rate of growth in melanomas: characteristics and associations of rapidly growing melanomas. Arch Dermatol 2006; 142:1551-8. PubMed PMID: 17178980.

8. Lipsker D, Engel F, Cribier B, Velten M, Hedelin G. Trends in melanoma epidemiology suggest three different types of melanoma. Br J Dermatol. 2007 Aug; 157:338-43. PubMed PMID: 17596175.

9. Cañueto J, Martín-Vallejo J, Cardeñoso-Álvarez E, Fernández-López E, Pérez-Losada J, Román-Curto C. Rapid growth rate is associated with poor prognosis in cutaneous squamous cell carcinoma. Clin Exp Dermatol. 2018 Dec;43:876-882. doi: 10.1111/ced.13570.

10. Kricker A, Armstrong B, Hansen V, Watson A, Singh-Khaira G, Lecathelinais C, Goumas C, Girgis A. Basal cell carcinoma and squamous cell carcinoma growth rates and determinants of size in community patients. J Am Acad Dermatol. 2014 Mar;70:456-64. PubMed PMID: 24373780.

11. Eide MJ, Weinstock MA, Dufresne RG Jr et al. Relationship of treatment delay with surgical defect size from keratinocyte carcinoma (basal cell carcinoma and squamous cell carcinoma of the skin). J Invest Dermatol. 2005 Feb;124:308-14. PubMed PMID: 15675948.

12. Renzi C, Mastroeni S, Passarelli F et al. Factors associated with large cutaneous squamous cell carcinomas. J Am Acad Dermatol. 2010 Sep;63:404-11. PubMed PMID: 
20598396.

This article is protected by copyright. All rights reserved. 


\section{Figures legends}

Figure 1: a-b) Primary cutaneous squamous cell carcinoma (cSCC) located on the right parietal-occipital scalp region; c-d) Recurrence of cSCC.

Figure 2: a-c) Rapidly progressing growing subcutaneous metastases.

Figure 3: Timeline. 


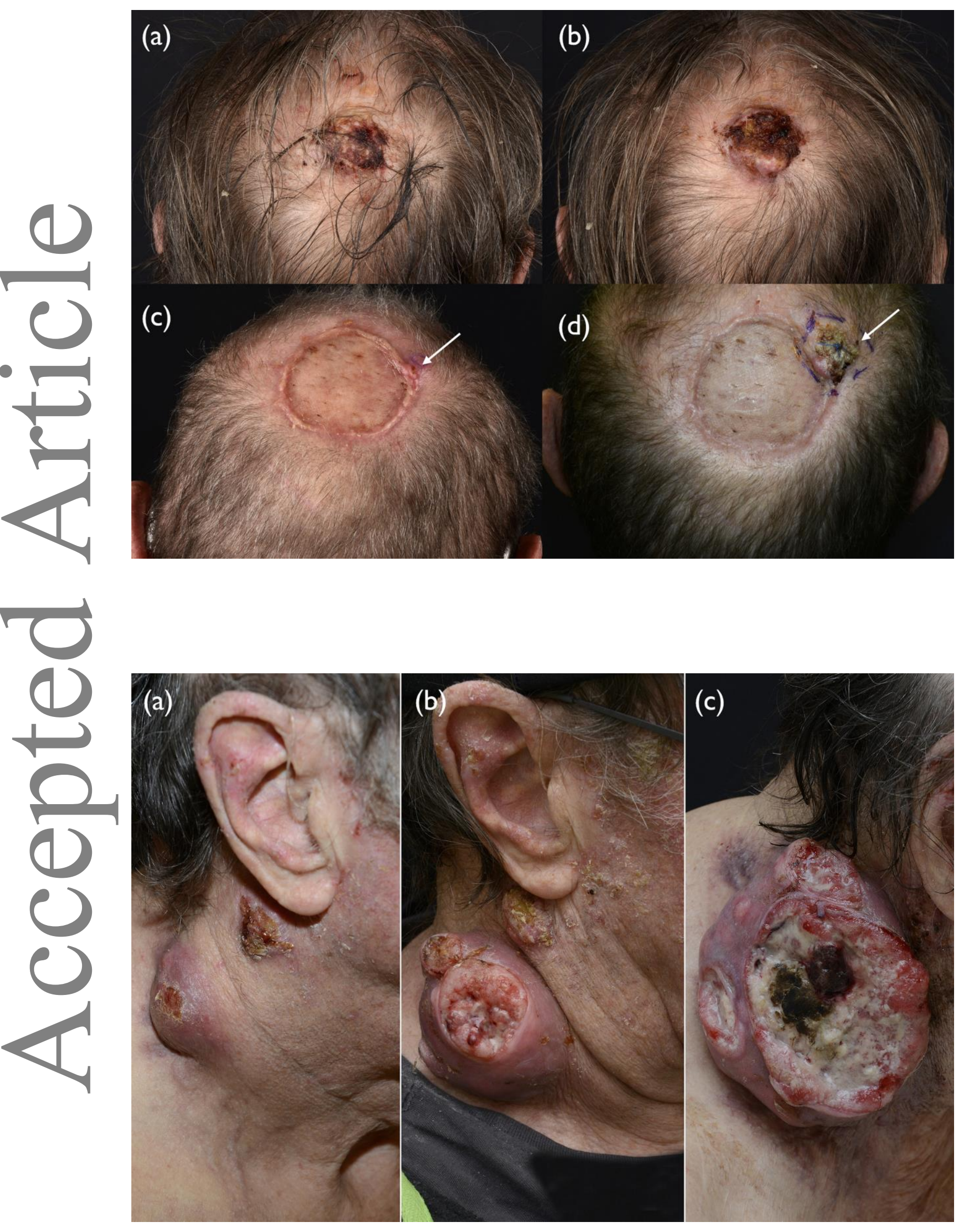




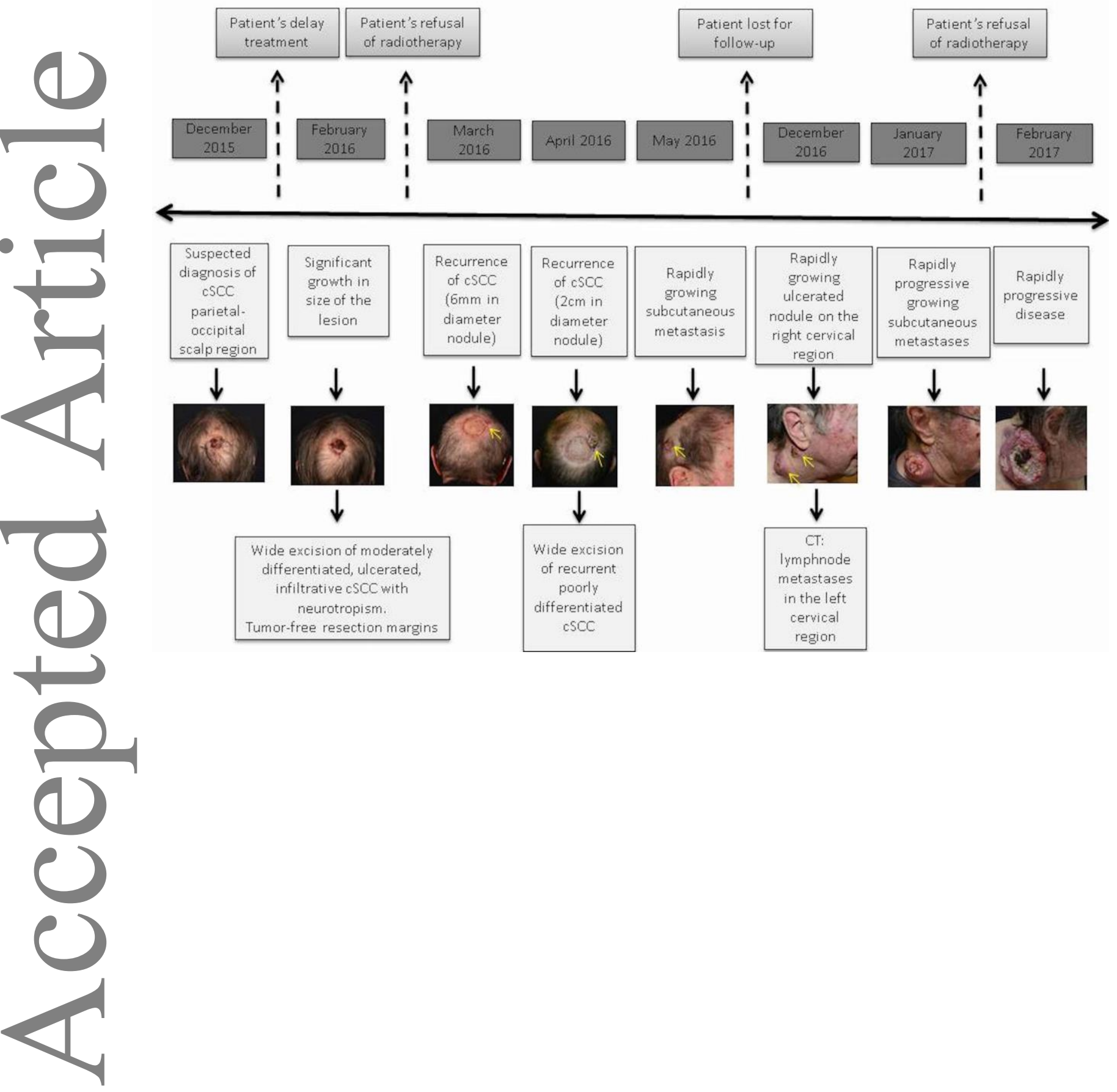

